\title{
An integrated approach to computer usage in the teaching of experimental psychology
}

\author{
STEPHEN C. HIRTLE and HOWARD J. KALLMAN \\ State University of New York at Albany, Albany, New York
}

\begin{abstract}
Ongoing development of a series of laboratory modules for experimental psychology courses is described. The modules are designed to be implemented on a network of TRS-80 microcomputers, which are linked by a Network 3 controller. Within each module, the computer controls stimulus events, records individual data, probes the student for understanding of experimental concepts, and tabulates the class data. In addition to a discussion of the modules and their software implementation, the required hardware is described.
\end{abstract}

There has been a recent increase in microcomputerbased instruction in undergraduate courses, particularly in laboratory courses. However, much of this work has focused on using the computer for such things as presenting stimuli, collecting responses, and performing statistical analyses. Although such computer usage clearly represents an improvement over the use of such material as $3 \times 5$ cards as laboratory instrumentation, it does not capitalize on the potential of the computer as a "smart" machine (Arons, 1984; Larkin, 1983). Given this limitation, there appears to be a need to develop interactive computer material that can be used in experimental psychology courses (Eckerman, 1981; Spivey, 1983). It was our desire to develop such materials for use in our experimental psychology courses at SUNY-Albany.

The first problem that we had to address concerned implementation. Some existing software, although suitable for single machines, had been cumbersome to use within a full-scale laboratory course in which hundreds of students were registered in over a dozen small sections under the guidance of several instructors and teaching assistants. The option of networking is an efficient, but often expensive, solution. However, there are a few low-cost networks available. One option that we explored was the possibility of using a low-cost multiuser system based on TRS-80 Model 3 microcomputers.

After obtaining the necessary funding for the purchase of the required hardware, we began development of a series of modules for experimental psychology for implementation on the network of TRS-80 microcomputers. The modules were designed to both control experiments and probe for understanding of the experimental concepts relevant to the experiment. These components have been integrated into single programs, which can be run on a

This project was supported in part by a Faculty Grant for the Improvement of Undergraduate Instruction for the Research Foundation of the State University of New York awarded to both authors. We would like to thank Shirley Brown, Gary Rockwood, and Joe DeCola for their comments on the programs and their assistance in the implementation of this project. Correspondence should be addressed to the above authors at the Department of Psychology, 1400 Washington Avenue, Albany, NY 12222 .
TRS-80 Model 3 or Model 4 microcomputer and can be completed during a 1- or 2- $\mathrm{h}$ lab session. Although simpler versions of the programs can be run on isolated stations, the modules were written for use with a Network 3 controller, which allows the monitoring of 16 student stations at one time. The network host station can record each student's progress and flag students that are in need of additional (personal) help. A general outline of our approach and a specific example of one module are detailed below.

\section{GENERAL OUTLINE}

A series of programs are being developed for use in 1- or 2-h weekly lab sections of an introductory course in experimental psychology. The programs constitute limited replications of both classic and contemporary psychology experiments. The student acts as both experimenter and subject. At the beginning of the experiment, the computer asks the student to enter a few initial parameters, after which the computer administers the experimental conditions and the student responds accordingly. After completion of an experiment, the studentgenerated data are sent to the host computer and then printed out for later use (in student lab reports, etc.). This framework has been adopted by others for use in experimental laboratory courses (Bewley, 1979; Perera, 1981), although most previous work has not made use of a computer network.

We have expanded this basic framework, so that in addition to the simple running of an experiment during the lab session, the students participate in probe sequences at specified intervals during the course of the experiment to assess their understanding of key concepts. For example, at critical points in the experiment, the students are asked questions about the experimental design, significance of particular experimental manipulations, etc. Performance of the students is monitored by the host computer, both for evaluation purposes and for identification course topics that merit further discussion in the lecture part of the course. Furthermore, the program provides guidance to students who have difficulty with specific 
probe questions. Feedback to the lab instructor on "problem" concepts is immediate, and, thus, the instructor's closing comments for the lab section can accurately reflect the students' experiences and difficulties during the just-completed lab.

\section{HARDWARE}

The system is based on the use of a Network 3 controller. Up to 16 TRS-80 Model 3 computers can be connected to a single host computer through the network device. As a minimum, the host computer must have $32 \mathrm{~K}$ of memory and one floppy disk drive, and each of the student computers must have $16 \mathrm{~K}$ of memory. No disk drives are required for the student computers. All communication between computers is done through RS-232C serial ports. These ports are not standard on TRS-80 Model 3 computers and need to be installed on all computers as options.

Our configuration is expanded slightly over these minimums to include $48 \mathrm{~K}$ of memory on all computers and two disk drives in the host machine. To avoid cassette loading of the network operating system in each student machine, we also purchased optional ROM chips for each student machine, which facilitate the loading of the Network 3 software. All printing is done on a dot matrix printer, which is interfaced with the host computer via a parallel port. At current retail prices, the cost of the entire system would be between $\$ 15,000$ and $\$ 20,000$. Educational discounts, if available, would lower the cost.

Any BASIC program in the Radio Shack dialect can be run at the 16 student stations, so long as it does not exceed the available memory on the student station. The network operating system and network BASIC (NBASIC) use 9K of RAM in each student station, which is virtually identical to the memory load of TRSDOS disk BASIC. NBASIC contains many of the TRSDOS commands that are related to program execution, in addition to a few commands unique to a network, such as printer and disk selection. Up to 10 machine language subroutines can be stored and accessed in NBASIC. The student stations may all run the same program or as many as 16 different programs. Thus, the stations can be used independently as "smart terminals," in contrast with the less desirable option of networking "dumb terminals" (cf. Vom Saal, Eckerman, Balsam, \& McDaniel, 1984). The host station is dedicated to supervising the system during operation.

The printer provides immediate feedback about the progress of each student through messages and bell signals, and the disk drives at the host station provide permanent storage of progress reports, data, or queries. The programs described below make active use of both the printer and the disks.

\section{USE OF THE NETWORK}

\section{Computer Integration}

Although our project included a number of lab mod- ules in which the computer assists in all functions of a lab session, we also felt a need for modules that were integrated with traditional stimulus materials, and thus, our first modules were developed along these lines. One module allows the computer to function as a stopwatch by using a simple looping algorithm (Perera, 1979). The same module will then prompt the subject for data entry, and the data are sent via the network to the host disk. When all the students in the lab are finished, the instructor runs a supplementary program that prints copies of the class data for distribution at the end of the class. At the same time, simple analyses, such as calculating class means, regression coefficients, or statistical tests, can be carried out and reported to the class for general information, held by the instructor to check lab write-ups, or used for immediate evaluation of the success of the lab.

Even for labs that do not inherently require a computer, such as running through various Stroop conditions, we have found the networked computers to be useful as a stopwatch and as a data storage and analysis mechanism. By using the network, we were able to tabulate and store data from 16 independent lab sections for a total of $256 \mathrm{stu}$ dents over the course of a week, without difficulties.

\section{Computer-Assisted Experimentation}

The next area of application that we explored was the development of computer-assisted experimentation (CAE), in which the computer would present stimuli, collect responses, and tabulate data. There are several sources of CAE on the market or in the public domain for independent systems, such as the Apple II (Bewley, 1979; Poltrock \& Foltz, 1982), the PET (Simpson \& Burgess, 1984), and the TRS-80 (Bewley, 1979; Perera, 1981). With the exception of translating one BASIC version to another, there is little problem in upgrading these programs to take advantage of the network, so that at key points in the lab, data can be sent to the host disk drives and a progress report can be sent to the printer.

\section{Computer-Assisted Instruction}

A third area of application was to use the computer to assist in instructional dialogs. Arons (1984) recently contrasted the "most gratuitous misuse of the computer," as a page turner of text material, with its potential use as the director of a Socratic dialog. In the former, the computer presents text and offers, at best, multiple-choice questions. The student is rarely engaged, but, rather, spends most of the time pressing the return key. A Socratic, or instructional, dialog, however, offers openended questions directed by the student's responses, and takes advantage of graphics, timing, and interactive displays to engage the student in the learning process (Arons, 1984).

One objective of our project has been to adapt a computer-assisted-instruction (CAI) format for use with the TRS-80 Model 3 network. Although others have used the TRS-80 for CAI activities in psychology courses (e.g., Balsam, Fifer, Sacks, \& Silver, 1984), it was our desire to integrate the CAI with the CAE activities. Given the 
nature of microcomputers, we were faced with several constraints. First, the only feasible language options were BASIC and assembly language. Thus far, we have done our programming in BASIC because it was not critical to have millisecond timing, but it was critical to have flexibility in updating the text portions of the program. Second, we needed to stay within the memory limitations of a microcomputer.

We overcame the constraints of language and memory by (1) restricting the domain of study, and by (2) using a keyword search/looping structure to direct the dialog, thus tracking a student's understanding through concepts mastered, rather than by the interpretation of protocols. Each of these points is discussed in order.

In order to be able to work within the memory capabilities of our computers, we restricted each program to specific topics, such as main effects versus interactions, or the use of scatter plots. In addition, each program is designed to complement a specific laboratory experiment. Thus, we have restricted the scope of the programs and in doing so have reduced the storage requirements.

Next, we introduced a keyword search to flag students' responses. Rather than parse sentences for meaning, simple answers are flagged for certain keywords or combinations of keywords. As the set of keywords is limited, there is a danger of the students' answers being correct and yet being missed by a flag. This problem is partially overcome by giving a "correct" answer after a small number of attempts that fail to be successfully flagged and by offering further clarifications after each unclear attempt. In addition, we record all answers for later "fine-tuning", of the programs.

By using a keyword search, we restrict the modeling of a student's understanding to the tracking of the concepts mastered by the student. It appeared that a more dynamic tracking technique, such as building a production system of declarative statements and condition-action rules (e.g., Larkin, 1983), was beyond the memory and language capabilities of a TRS-80 microcomputer. Our goal is to map the knowledge space of the class, where students are represented as points in the space. Our interests would then turn to describing the structure of the space and focusing on methods for efficiently locating a student within that space. For a complete discussion of knowledge spaces, see Doignon and Falmagne (1984) and Falmagne (1984).

\section{An Example: Decision Making and Interaction}

One concept that is difficult to demonstrate in experimental psychology is that of interactions between independent variables. One way to illustrate interactions (and individual differences) is through experiments on the role of risk in decision making (Reitman \& Meyer, 1980; Tversky \& Kahneman, 1981). Our laboratory project on decision making requires subjects on each trial to choose between a sure-thing (outcome of known value) and a risk (outcome of unknown value). The outcome of the surething has two values, negative or positive, whereas risk has three levels, high, medium, or low. Thus, the experiment is a $2 \times 3$ within-subjects design, with the dependent variable equal to the number of risks taken under each condition. A typical result shows a strong main effect for sure-thing, with more risks taken in the context of a negative sure thing, a small or nonexistent main effect for risk level, and an interaction between the two independent variables, with the effect of sure-thing being greater at low risk levels.

We have developed sequences that probe students' understanding of the methodology and appropriate data analysis of this experiment. These probe sequences represent part of the lab exercise in which subjects also run themselves through the conditions of the experiment. One probe sequence consists of an interactive data analysis segment in which students' suggestions for graphs and statistical analyses are presented and evaluated. The evaluation is based on clarity and simplicity, because students often suggest an "all possible analyses" option with little understanding of the conceptual issues involved. A second probe sequence explores the students' understanding of main effects and interactions, a topic that has received the attention of other CAI programs (e.g., Fazio \& Backler, 1983). An additional sequence being developed includes discussion of students' understanding of the design, with a focus on why a within-subjects design is beneficial when there are large individual differences, and how large individual differences may obscure the results (Mook, 1982). In all modules, a keyword-based analysis of responses directs the dialog, by flagging for several answers, and then constructing an appropriate answer using the students' wording whenever possible.

\section{Instructor's Role}

Throughout the implementation of these modules, the instructor plays a key role. Through messages to the printer, the instructor can monitor each student's progress and can assist individually those students having difficulty. Furthermore, the instructor's comments at the close of the session can reflect directly on the actual lab experiences.

\section{BENEFITS OF A NETWORK}

The use of the network of Radio Shack TRS-80 computers is a cost effective way to conduct exercises that allow both for the instructor to monitor individual students' progress during the lab and for students to access the data from all subjects in the class. The cost of the Network 3 controller and associated hardware and software is more than compensated for by the fact that the host computer is the only one that requires disk drives and a printer. With the network software installed, the speed of loading programs in all 16 computers ranges from 2 to $8 \mathrm{~min}$ for our programs. Although the use of a hard disk system would provide greater storage capabilities, we have not experienced any major storage difficulties using the floppy disks. Furthermore, by using a floppy disk-based 
system, we have access to more software that would be difficult to transport to a hard disk system (cf. Lowe, 1983).

\section{FUTURE DEVELOPMENT}

Our modules were designed with an eye on flexibility in order to facilitate future development. The experimental displays are designed using a framework that will allow future experiments to be programmed quickly in place of current ones. The probe sequences are designed to be flexible in that additional branches or alternative flowcharts can be added. Finally, the answers to the probe sequences are recorded, giving a permanent record of the logical chains used by students to answer to the probes. Their word-for-word answers can be used to fine-tune the probe process in future years and, at the same time, allow for the analysis of learning difficulties in a laboratory course.

\section{REFERENCES}

ARons, A. B. (1984). Computer-based instructional dialogs in science courses. Science, 224, 1051-1056.

Balsam, P., Fifer, W., Sacks, S., \& Silver, R. (1984). Microcomputers in psychology laboratory courses. Behavior Research Methods, Instruments, \& Computers, 16, 150-152.

BEWLEY, W. L. (1979). Cognitive psychology: A computer oriented laboratory manual. Wentworth, NH: Compress.

DoIGNON, J., \& FALMAGNE, J. (1984). The assessment of knowledge: Introduction to knowledge spaces. Manuscript in preparation.

ECKERMAN, D. A. (1981). Developing a laboratory course using PASCAL on the Apple II. Behavior Research Methods \& Instrumentation, 13, 204-208.
Falmagne, J. (1984, August). Introduction to knowledge spaces. Paper presented at the annual meeting of the Society for Mathematical Psychology, Chicago.

FAzIo, R. H., BACKLER, M. H. (1983). Computer lessons for a social psychology research methods course. Behavior Research Methods \& Instrumentation, 15, 135-137.

LARKIN, J. H. (1983). A general knowledge structure for learning or teaching science. In A. C. Wilkinson (Ed.), Classroom computers and cognitive science (pp. 53-70). New York: Academic Press.

LowE, D. C. (1983). A Winchester hard-disk integrated computerassisted instructional laboratory: Hardware and data management considerations. Behavior Research Methods \& Instrumentation, 15 181-182.

Moox, D. G. (1982). Psychological research: Strategy and tactics. New York: Harper \& Row.

Perera, T. B. (1979). A comparison of BASIC language timing loops for the TRS-80 microcomputer. Behavior Research Methods \& Instrumentation, 11, 592

Perera, T. B. (1981). A laboratory in experimental psychology using the TRS-80. Behavior Research Methods \& Instrumentation, 13 195-197.

Poltrock, S. E., d Foltz, G. S. (1982). An experimental psychology laboratory system for the Apple II microcomputer. Behavior Research Methods \& Instrumentation, 14, 103-108.

ReITMAN, J. S., \& MEYER, D. (1980). Superlab, as a natural science. Unpublished laboratory manual, University of Michigan.

SimPSON, G. B., \& BURGESs, C. (1984). PET programs for demonstration and student research in cognitive psychology. Behavior Research Methods, Instruments, \& Computers, 16, 65-66.

SPIVEY, J. E. (1983). Software development for computer-assisted instruction in experimental psychology. Behavior Research Methods \& Instrumentation, 15, 183-186.

TVersKy, A., KahNEman, D. (1981). The framing of decisions and the psychology of choice. Science, 211, 453-458.

Vom Saal, W., Eckerman, D. A., Balsam, P., \& McDaniel, C. (1984). Getting started with microcomputers in undergraduate education: Hints and guidelines. Behavior Research Methods, Instruments, \& Computers, 16, 65-66. 